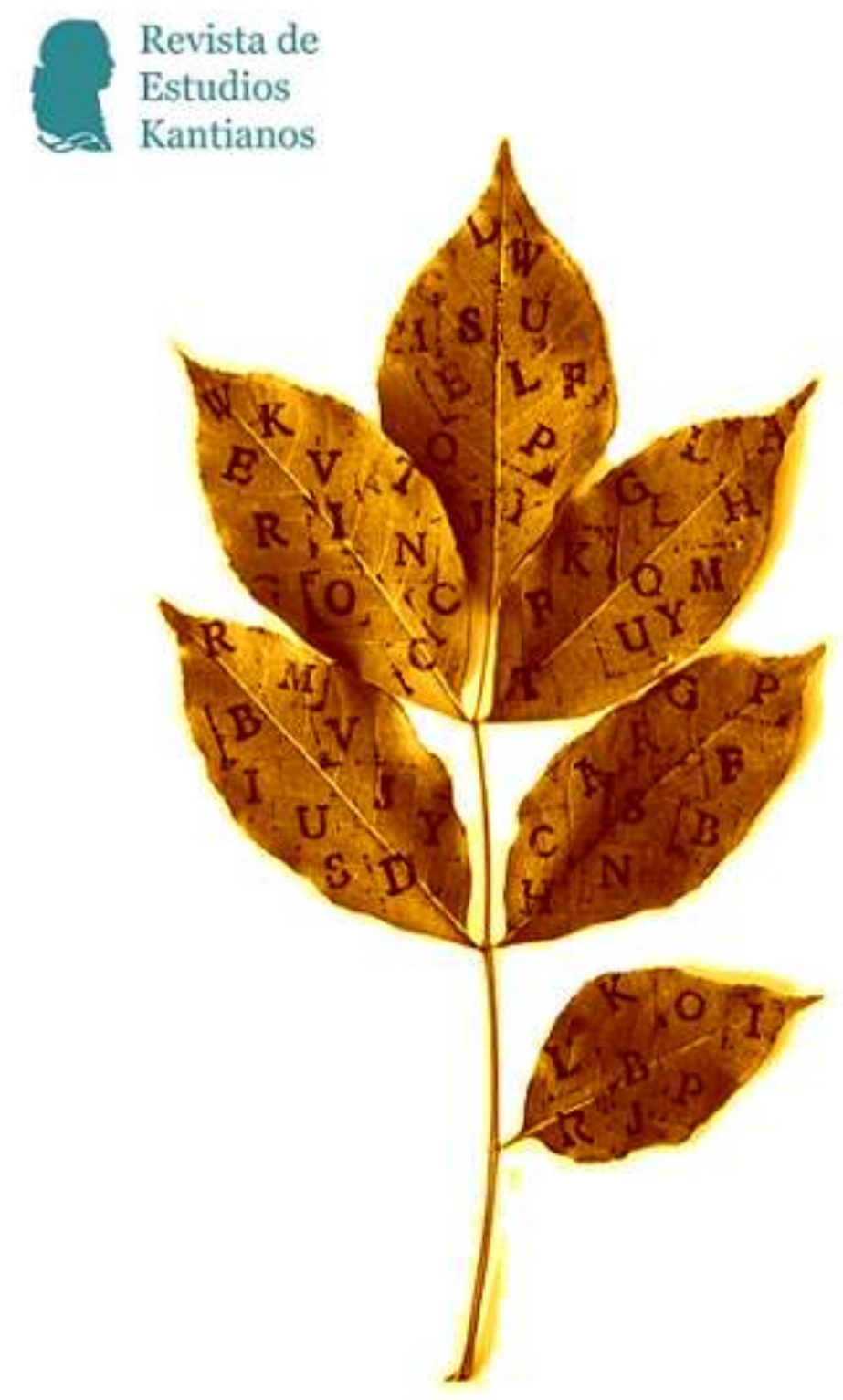




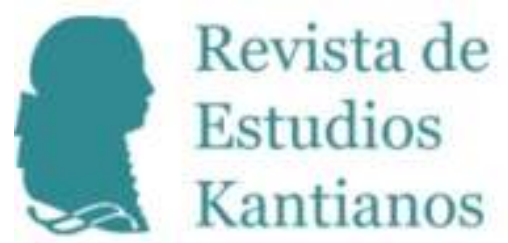




\section{Revista de Estudios Kantianos}

Publicación internacional de la Sociedad de Estudios Kantianos en Lengua Española Internationale Zeitschrift der Gesellschaft für Kant-Studien in Spanischer Sprache International Journal of the Society of Kantian Studies in the Spanish Language

\section{Dirección}

Pedro Jesús Teruel, Universitat de València

pedro.teruel@uv.es

Hernán Pringe, CONICET-Universidad de Buenos Aires/

Universidad Diego Portales, Santiago de Chile

hpringe@gmail.com

\section{Secretario de edición}

Fernando Moledo, Universidad de Buenos Aires - CONICET

fernandomoledo@gmail.com

\section{Secretaria de calidad}

Marcela García, Instituto de Investigaciones Filosóficas, UNAM

garciar.marcela@gmail.com

\section{Editores científicos}

Jacinto Rivera de Rosales, UNED, Madrid

Claudia Jáuregui, Universidad de Buenos Aires

Vicente Durán, Pontificia Universidad Javeriana, Bogotá

Julio del Valle, Pontificia Universidad Católica del Perú, Lima

Jesús Conill, Universitat de València

Gustavo Leyva, Universidad Autónoma de México, México D. F.

María Xesús Vázquez Lobeiras, Universidade de Santiago de Compostela

Wilson Herrera, Universidad del Rosario, Bogotá

Pablo Oyarzun, Universidad de Chile, Santiago de Chile

Paula Órdenes Azúa, Universität Heidelberg 


\section{Comité científico}

Juan Arana, Universidad de Sevilla

Reinhardt Brandt, Philipps-Universität Marburg

Mario Caimi, Universidad de Buenos Aires

Monique Castillo, Université de Paris-Est

Adela Cortina, Universitat de València

Bernd Dörflinger, Universität Trier

Norbert Fischer, Universität Eichstätt-Ingolstadt

Miguel Giusti, Pontificia Universidad Católica del Perú

Dulce María Granja, Universidad Nacional Autónoma de México

Christian Hamm, Universidad Federal de Santa María, Brasil

Dietmar Heidemann, Université du Luxembourg

Otfried Höffe, Universität Tübingen

Claudio La Rocca, Università degli Studi di Genova

Juan Manuel Navarro Cordón, Universidad Complutense, Madrid

Carlos Pereda, Universidad Nacional Autónoma de México

Gustavo Pereira, Universidad de la República, Uruguay

Ubirajara Rancan de Azevedo, Universidade Estadual Paulista, Brasil

Margit Ruffing, Johannes Gutenberg-Universität Mainz

Gustavo Sarmiento, Universidad Simón Bolívar, Venezuela

Sergio Sevilla, Universitat de València

Roberto Torretti, Universidad Diego Portales, Santiago de Chile

Violetta Waibel, Universität Wien

Howard Williams, University of Aberystwyth

Allen W. Wood, Indiana University

Diseño, revisión de estilo y maqueta

Josefa Ros Velasco, Universidad Complutense de Madrid

\section{Entidades colaboradoras}

Sociedad de Estudios Kantianos en Lengua Española (SEKLE)

Departament de Filosofia de la Universitat de València 


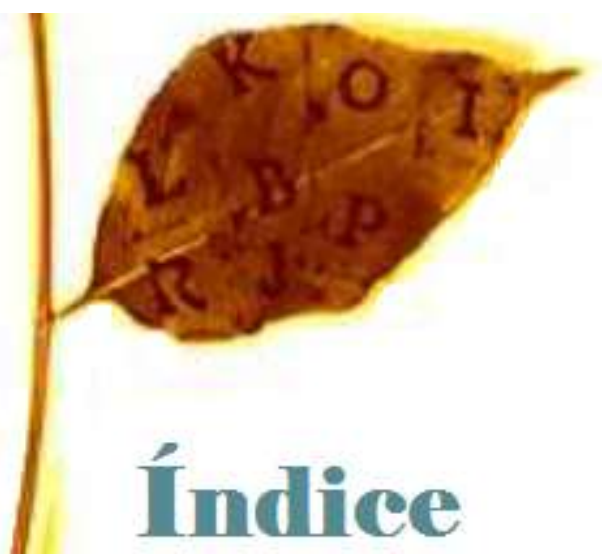

\section{Artículos}

113 ¿"El yo propiamente tal” (I. Kant) o "el originario ser sí mismo” (D. Heinrich)? Sobre algunas características del concepto kantiano de autoconciencia

Heiner Klemme

DOI 10.7203/REK.2.2.9068

126 La noción kantiana de verdad transcendental

Stéfano Straulino Torre

DOI 10.7203/REK.2.2.8809

146 Una travesía kantiana a través del Escila constructivista y el Caribdis realista. Apuntes para un abordaje kantiano-constitutivista de las fuentes de la normatividad

Martín Fleitas González

DOI 10.7203/REK.2.2.8810

174 El malestar kantiano. Filosofía y ciencia al encuentro con la naturaleza Juan Felipe Guevara

DOI 10.7203/REK.2.2.8812

191 ¿Puede haber una fundamentación kantiana de los derechos humanos? Algunas consideraciones críticas

Nuria Sánchez Madrid

DOI 10.7203/REK.2.2.8871

\section{Semblanza}

208 El pensamiento filosófico de Ernesto Mayz Vallenilla

Gustavo Sarmiento

DOI 10.7203/REK.2.2.9021 


\section{Reseñas}

217 Claudia Jáuregi, Fernando Moledo, Hernán Pringe y Marcos Thisted (eds.) Crítica y Metafísica. Homenaje a Mario Caimi. Studien und Materialen zur Geschichten der Philosophie. Hildesheim, Olms, 2015, 460 pp. ISBN: 978-3-487-15237-0.

Alba Jiménez Rodríguez.

DOI 10.7203/REK.2.2. 8814

227 Fernando Moledo: Los años silenciosos de Kant: Aspectos de la génesis de la Deducción Trascendental en la década de 1770. Seguido de la traducción del Legado de Duisburg (ca. 1775). Buenos Aires, Prometeo, 2014, 192 pp. ISBN: 978-987-574-667-1.

Pablo Moscón DOI 10.7203/REK.2.2. 8830

231 Salvi Turró: Filosofia i Modernitat. La reconstrucció de l'ordre del món. Barcelona, Edicions Universitat de Barcelona, 2016, 228 pp. ISBN: 978-84-475-3966-6.

Pedro Jesús Teruel

DOI 10.7203/REK.2.2. 8887

233 Gabriel Rivero: Zur Bedeutung des Begriffs Ontologie bei Kant. Berlín, Boston, Walter de Gruyter, 2014, 247 pp. ISBN: 978-3-11-034180-5.

Luciana Martínez

DOI 10.7203/REK.2.2.8881

Informe

237 Informe sobre el III Congreso de la Sociedad de Estudios Kantianos en Lengua Española (SEKLE)

Luciana Martínez

DOI 10.7203/REK.2.2.9011

\section{Convocatoria}

242 La recepción de Kant en México

Dulce María Granja Castro; Gustavo Leyva Martínez

Eventos y normas para la publicación

244 Noticias y normas para autores 


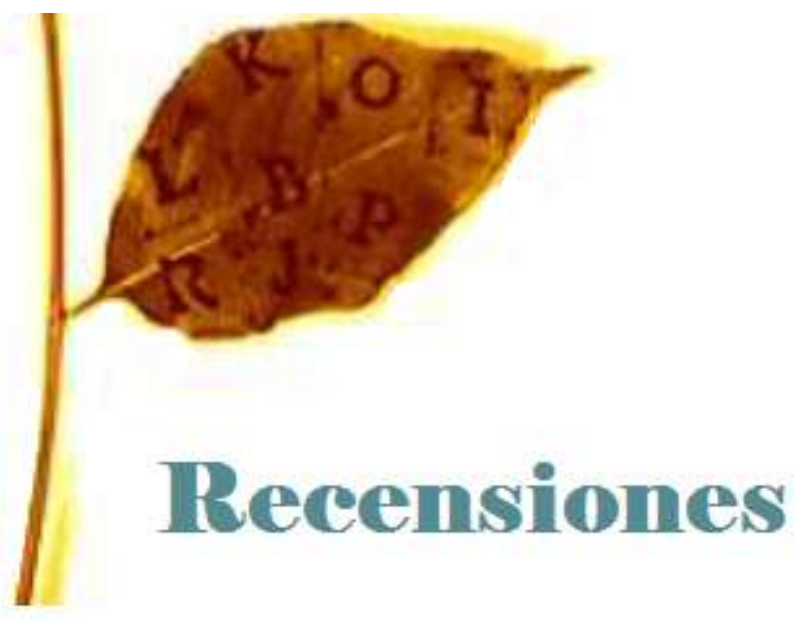





\title{
Salvi Turró: Filosofia i Modernitat. La reconstrucció de l'ordre del món. Barcelona, Edicions Universitat de Barcelona, 2016, 228 pp. ISBN: 978- 84-475-3966-6.
}

\author{
PEDRO JESÚS TERUEL ${ }^{1}$
}

Son significativas las aportaciones de Salvi Turró a una mejor comprensión de la modernidad filosófica. Entre ellas se encuentran las monografías Descartes. Del hermetismo a la nueva ciencia (1985), Tránsito de la naturaleza a la historia en la filosofía de Kant (1996), Lliçons sobre història i dret a Kant (1997) y Fichte. De la consciència a l'absolut (2011), así como numerosos artículos en revistas científicas. El presente volumen reúne nueve de estos últimos -revisados, reelaborados y, en su caso, traducidos- junto con un texto inédito. El resultado es prenda del estilo del autor: sólido en la fundamentación, eficaz en la exposición, nunca banal en la elección de temas y enfoques.

La noción de 'mundo' en torno a la cual gira el subtítulo (La reconstrucción del orden del mundo) aparece aquí, a mi juicio, en al menos cuatro perspectivas complementarias. Se trata (a) del conjunto de elementos y rasgos ónticos y metafísicos que caracterizan lo real en el pensamiento clásico y medieval; en segundo lugar, (b) del conjunto de condiciones transcendentales y ontológicas que ocupan el lugar de aquéllos en la filosofía moderna. Un modelo y otro dibujan (c) horizontes conceptuales heterogéneos para el abordaje de la pregunta sobre la unidad de lo real y su sentido, ya sea considerado en su globalidad o en relación con el ser humano. En cuarto lugar, (d) el tránsito desde (a) hacia (b) da pie al establecimiento de pautas cronológicas y temáticas en el seno de la historia de la filosofía y, con ellas, a la delimitación respectiva de 'lo moderno' frente a 'lo medieval', por un lado, y frente a 'lo postmoderno', por otro. Pues bien: la presente obra trata fundamentalmente de las perspectivas segunda y tercera, con una incursión en la cuarta; todo ello, sobre el trasfondo del abandono histórico de la primera perspectiva.

La cubierta del libro reproduce el fascinante grabado Melancolía I, de Alberto Durero (1514). La alada figura que lo protagoniza, acuclillada con ademán indolente entre tablas matemáticas, instrumentos de medida y herramientas de trabajo, lanza una mirada nostálgica fuera de campo. Es la añoranza de la unidad del mundo - de ese mundo que se esfuma, disuelto por reducciones metodológicas y desplazamientos ontológicos, a caballo entre los siglos XVI y XVII- la que lleva a pergeñar múltiples proyectos de reconstrucción. Y éstos constituyen el hilo conductor de Filosofia i Modernitat.

La tesis de fondo, explicitada en la introducción, apunta a dicha multiplicidad. Lejos de constituir un bloque homogéneo de propuestas sobre la reconstrucción del orden del mundo -en los marcos trazados por las perspectivas (b) y (c)-, la Modernidad cobija esquemas conceptuales variados y a menudo opuestos entre sí. En la primera parte del volumen ("La filosofía moderna: concepto y perspectivas") se presentan diversas vertientes de dicha heterogeneidad: el modo en que la reducción mecanicista coadyuva al desarrollo del dominio técnico como uno de los rasgos distintivos de la mentalidad moderna; la vinculación del nuevo paradigma con el vaciamiento de sentido del libro de la Naturaleza y del libro de la Revelación, a instancias, respectivamente, de su lectura en clave ateleológica e histórico-crítica; la presencia viva en la mentalidad moderna de transformaciones conceptuales fecundadas por el cristianismo, expuestas aquí al hilo de las nociones de 'creación', 'libre albedrío' y 'encarnación'; el papel teórico de la intersubjetividad en el trayecto que media entre Descartes y Hegel. Cada análisis corresponde a un capítulo de esta primera parte, que se inaugura con una incursión en la genealogía de la Modernidad como época al hilo de la cual se presenta distintos modelos de periodización.

${ }^{1}$ Universitat de València. 
La segunda parte ("La comprensión del mundo en la Modernidad") recoge otras cinco aportaciones. Dos de ellas giran en torno a Kant; se aborda ahí, concretamente, el tópico de la sabiduría del mundo y su enlace con la filosofía de la historia. En la tercera se analiza el tránsito de la filosofía kantiana a la fichteana, sobre el trasfondo de la distinción entre fenómeno y noúmeno y el papel de la subjetividad en su constitución respectiva. Fichte es el protagonista del cuarto capítulo, que pone de relieve el horizonte metafísico de la doctrina fichteana a partir de la noción de imagen $($ Bild $)$. En el último apartado se despliega un discernimiento crítico sobre la cercanía y la distancia entre las respectivas visiones del mundo en Hegel y Spinoza.

La urdimbre de la obra denota una mano experta. Turró ha cultivado una familiaridad con la red conceptual tejida entre Descartes y Hegel que le permite moverse con soltura en la reflexión sobre la génesis de la Modernidad y sus debates internos. Así se muestra, por ejemplo, en las excelentes contribuciones acerca de Kant; en la pequeña joya labrada en torno a la imagen del absoluto y las visiones del mundo en Fichte; o en el elaborado cotejo de Hegel y Spinoza. A mi juicio, hubiera sido deseable hacer hincapié en la faceta historiográfica. Se hubiese abonado así la cuarta perspectiva a la que me he referido supra y la vertiente de la tesis básica asociada a ella -a saber: que el pensamiento moderno transita por vías heterogéneas a la hora de emprender la reconstrucción eidética del orden del mundo, cosa que incide en el abordaje historiográfico y en la tarea ligada a la periodización-. Tratándose de un asunto largamente trabajado por el autor, nos quedamos con ganas de saber más sobre sus tomas de postura al respecto.

Concluiré subrayando algo que, por evidente, puede pasar desapercibido. Me refiero a la materialidad lingüística de la obra. Tras darle muchas vueltas al modo en que se habría de cultivar el catalán como lengua filosófica, Josep Ferrater Mora encabezaba la versión catalana de Philosophy Today con un aviso para navegantes: la relevancia científica de una lengua no proviene de indagaciones eruditas - de consumo doméstico- sobre su pedigrí histórico, sino de la capacidad de sus hablantes y escritores a la hora de plantear cuestiones significativas para el debate de las ideas (cf. $L a$ filosofia en el món d'avui, Barcelona, Edicions 62, 1965, 5-8). Salvi Turró es un navegante avezado y ha escrito esta obra para el mundo. Estudiosas y estudiosos de cualquier procedencia encontrarán en ella material rico y estimulante. Aprender una lengua para leer cosas que merecen la pena depara un gozoso placer intelectual; en este sentido, el presente volumen ofrece un aliciente más para acercarse a la hermosa lengua catalana. 